\title{
Impact of assessment of bone status before corrective surgery of lumbar spine in patients over 50 years old
}

This article was published in the following Dove Press journal: Open Access Rheumatology: Research and Reviews

\author{
Christopher Banse ${ }^{1,2}$ \\ Mourad Ould-Slimane ${ }^{3}$ \\ Emmanuel Foulongne ${ }^{3}$ \\ Alexis Perez ${ }^{4}$ \\ Gilles Avenel' \\ Alain Daragon' \\ Olivier Vittecoq' \\ 'Rheumatology Department, Rouen \\ University Hospital, 76000 Rouen, France; \\ ${ }^{2}$ Rheumatology Department, Polyclinique \\ Saint Côme, 60200 Compiègne, France; \\ ${ }^{3}$ Department of Orthopaedic Surgery, \\ Rouen University Hospital, 76000 Rouen, \\ France; ${ }^{4}$ Department of Neurosurgery, \\ Rouen University Hospital, 76000 Rouen, \\ France
}

Background and objective: There is absence of data on the prevalence of osteoporosis before corrective surgery of the lumbar spine. We do not know the impact of bone assessment before corrective spine surgery, regarding the prevalence of osteoporosis, risk factors for osteoporosis, and prescription of osteoporotic treatment. Our objective was to evaluate the impact of assessment of bone status before corrective surgery of the lumbar spine.

Methods: This retrospective study was conducted over a period of 30 months. Patients included were over 50 years old and had been referred to rheumatology consultation prior to corrective surgery of the lumbar spine with osteosynthesis, for scoliosis or spondylolisthesis. Assessment of bone status consisted in looking for risk factors for osteoporotic fracture, performing bone densitometry with the calculation of TBS (trabecular bone score) and the possible introduction of treatment for osteoporosis. Data were collected on complications related to bone fragility during follow-up.

Results: Twenty-eight patients with a median age of 71.2 years (55.5-84.8) were included; $89 \%$ were women. T score was $<-2.5$ in $14.3 \%(4 / 28)$ and -1 to -2.5 in $42.9 \%(12 / 28)$ on at least one of the three sites analyzed. Fifty percent of patients had a TBS $<1.2$, a history of more than four falls per year, a duration of more than $20 \mathrm{~s}$ in the Timed Up and Go Test, and/ or sedation treatment. Vitamin-calcium supplementation and treatment for osteoporosis were prescribed in $71.4 \%$ and $17.8 \%$ of cases, respectively. During follow-up, 3 patients had one or more osteoporotic vertebral fractures and 4 patients had loosening of implanted devices.

Conclusion: Despite a low prevalence of densitometric osteoporosis and therapeutic management, one in four patients had a bone complication, suggesting the superiority of TBS as an indicator of bone status

Keywords: osteoporosis, spine surgery, bone evaluation, fracture, TBS

\section{Introduction}

With the aging of the population, spine surgeons have to operate older patients with bone fragility associated with osteoporosis. ${ }^{1}$ Currently, preoperative assessment includes an evaluation by anesthetists, and sometimes by geriatric physicians, to assess the nutritional, cognitive, and functional status of elderly patients. This assessment can help identify patients at risk for perioperative and postoperative complications. ${ }^{2}$ In this context, before the introduction of implanted devices in the lumbar spine, it could be interesting to assess bone status, and particularly the risk of osteoporotic fracture and loosening of implanted devices. ${ }^{3}$
Correspondence: Christopher Banse Department of Rheumatology, Rouen University Hospital, I rue de Germont 7603I Rouen cedex, France

Tel +3 3682982749

$\mathrm{Fax}+33232880439$

Email totobanse@orange.fr 
The objectives of our study were, on the one hand, to determine the frequency of osteoporosis and its therapeutic consequences (indication or not for osteoprotective therapy) in patients requiring corrective surgery of the spine, and on the other hand, to collect data on fracture complications and/or loosening of implanted devices within 2 years after the surgical procedure.

\section{Methods}

This retrospective and longitudinal study was carried out at Rouen University Hospital between February 2015 and August 2017. Patients included were $>50$ years old and had been referred for a rheumatology consultation for assessment of bone status before corrective surgery of the lumbar spine (scoliosis and spondylolisthesis) with placement of screw. Data collected were: demographic characteristics (age, sex, body mass index, and history of lumbar spine surgery), risk factors for osteoporosis, of falling, and osteoporotic fractures. During the consultation, data on several parameters were collected: the calculation of daily calcium intake, the calculation of the percentage of probability of major fracture at 10 years using the Fracture Risk Assessment Tool (FRAX) score (in the absence of a $\mathrm{T}$ score less than -3 and osteoporotic fracture), results of biology tests (serum calcium, blood protein electrophoresis, serum $25 \mathrm{OH}$ vitamin $\mathrm{D}$, blood count, renal function, and liver enzymes) and results of bone densitometry tests using a Hologic Horizon Wi device ( $\mathrm{T}$ score for the lumbar spine, femoral neck and/ or ultra-distal radius as well as calculation of the trabecular bone score). At the end of the bone assessment, the rheumatologist decided on the initiation or not of osteoprotective therapy independently of the indication for spine surgery and in the absence of specific recommendations. ${ }^{4}$ The characteristics related to spine surgery were: the duration of spinal symptomatology, the maintenance of operative decision, and the search for osteoporotic fractures and/or loosening of implanted devices, occurring within 2 years after the intervention. Patients were followed in consultations by the surgeon who had operated.

Results are presented as medians with lower and upper limits or as percentages.

The study was approved by the noninterventional ethics committee of Rouen University Hospital (E201801). Since the study was retrospective, carried out from data collected according to a standardized procedure in usual care, no patient consent is required at the Rouen
University Hospital. Only the agreement of the Ethics Committee for retrospective noninterventional studies is sufficient. Declaration of Helsinki was followed in respect to privacy and confidentiality of patient data.

\section{Results}

\section{Demographic characteristics}

Spine surgeons at Rouen University Hospital referred 28 patients for rheumatology consultation over a period of 30 months. Their characteristics are summarized in Table 1. The median age was 71.2 years $(55.5-84.8)$ and $89 \%$ were women. The median body mass index was 30.7 $(19.6-50.7) \mathrm{kg} / \mathrm{m}^{2} ; 32 \%$ of patients had previously undergone surgery of the lumbar spine.

\section{Assessment of bone status}

In our population, risk factors for osteoporosis were: active smoking in $28.6 \%(8 / 28)$, early menopause ( $<40$ years) in $17.8 \%(5 / 28)$, corticosteroid therapy (more than $7.5 \mathrm{mg}$ /day

Table I Demographic data and assessment of bone condition prior to surgical correction of the spine in 28 patients

\begin{tabular}{|l|l|}
\hline Demographic characteristics & \\
\hline Age, years & $71.2(55.5-84.8)$ \\
Women, $\mathrm{n}(\%)$ & $25(89 \%)$ \\
Body mass index (kg/m²) & $30.7(19.6-50.7)$ \\
History of lumbar spine surgery, $\mathrm{n}(\%)$ & $9(32 \%)$ \\
\hline Risk factors for osteoporotic fractures & \\
Tobacco, $\mathrm{n}$ (\%) & $8(28.6 \%)$ \\
Early menopause, $\mathrm{n}(\%)$ & $5(17.8 \%)$ \\
Personal history of osteoporotic fractures, $\mathrm{n}(\%)$ & $4(14.3 \%)$ \\
Decreased visual acuity, $\mathrm{n}(\%)$ & $3(10.7 \%)$ \\
History of falls in the previous year, $\mathrm{n}(\%)$ & $14(50 \%)$ \\
Daily sedation therapy & $14(50 \%)$ \\
(anxiolytic/psychotropic), $\mathrm{n}(\%)$ & \\
"Get up and go test" (time in seconds) & $17(10-5 \mathrm{I})$ \\
Daily calcium intake in mg & $714(337-1889)$ \\
25 OH vitamin D serum level in ng/mL & $24(4.5-72)$ \\
Carboxy-terminal telopeptide collagen serum & $0.415(0.14-\mathrm{I})$ \\
level in ug/L & \\
T score $\leq-2.5, \mathrm{n}(\%)$ & $4(14.3 \%)$ \\
T score <-I to <-2.5, $\mathrm{n}(\%)$ & $12(42.9 \%)$ \\
TBS (trabecular bone score) & $1.213(1.04-1.46)$ \\
\hline Therapeutic consequences (before surgery) & \\
Calcium supplementation, $\mathrm{n}$ (\%) & $6(21.4 \%)$ \\
Vitamin D supplementation, $\mathrm{n}$ (\%) & $18(64.3 \%)$ \\
Zoledronic acid, $\mathrm{n}$ (\%) & $4(14.3 \%)$ \\
Denosumab, $\mathrm{n}$ (\%) & $\mathrm{I}(3.6 \%)$ \\
\hline
\end{tabular}

Note: Results are expressed in median (extremes) unless indicated otherwise. 
prednisone equivalent), and rheumatoid arthritis in one case (Table 1). Risk factors for osteoporotic fracture were: a history of falls (4 falls per year) in the previous year and sedation treatment in $50 \%(14 / 28)$, a personal history of major osteoporotic fracture in $14.3 \%(4 / 28)$, a decrease in visual acuity in $10.7 \%(3 / 28)$, and a history of fracture of the upper extremity of the femur in first degree relatives. The median duration of the timed-up-and-go test (TUG) was $22.56(10-51) \mathrm{s}$ with a score $>20 \mathrm{~s}$ in $44 \%$ of cases $(11 / 25)$. Median daily calcium intakes were estimated at $714 \mathrm{mg}$ (337-1889). Median hydroxy-vitamin D assay was 24 (4.5-72) $\mathrm{ng} / \mathrm{mL} \quad(\mathrm{N}: \quad 30-80 \quad \mathrm{ng} / \mathrm{mL})$. The median C-telopetide dosage of collagen was $0.415(0.14-1) \mu \mathrm{g} / \mathrm{L}$ (N: 0.33-0.88). The results obtained during the rheumatology consultation detected hyperparathyroidism in two patients and monoclonal gammapathy of undetermined significance in one patient. The median T scores at the lumbar spine $(n=17)$, femoral neck $(n=27)$, and at the lower end of the radius $(\mathrm{n}=10)$ were $-0.6(-2.9 ;+3.8),-1(-2.6 ;+1.3)$, and $-0.85(-3.1 ;+1.5)$, respectively. Four patients $(14.3 \%)$ had a $\mathrm{T}$ score of $\leq-2.5$ and 12 patients $(42.9 \%)$ had osteopenia (T score $<-1$ to $<-2.5$ ) on at least one of the three sites analyzed. The median trabecular bone score (TBS) was 1.213 (1.04-1.46). Based on the current recommendations, ${ }^{4}$ six patients were on osteoprotective treatment, four of whom had a history of major fractures, one patient had a T score $<-$ 3 , and one patient was above the threshold for therapeutic intervention after calculation of the FRAX score. ${ }^{5}$ The decision to introduce calcium and vitamin D supplementation was applied in 6 and 18 patients, respectively. For four patients, zoledronic acid was introduced, while for one patient, denosumab was initiated. One patient refused osteoprotective treatment.

\section{Impact of bone assessment on surgical procedure}

Patients had surgery for static lumbar spine disorder (scoliosis, sagittal disorder) in $71 \%$ of cases and/or lumbar spondylolisthesis in $39 \%$ of cases. Among these patients, $75 \%$ had lumbar spinal stenosis. The surgical decision was maintained in $43 \%$ of cases ( 8 patients were lost due to comorbidities, 4 patients were lost to follow-up, and 3 patients were improved with medical treatment). The median time between surgical consultation and rheumatologic consultation was 84.5 (2-201) days. Four patients had loosening of their implanted device within 2 years of surgery. These patients had no osteoprotective treatment because they were not eligible according to current recommendations (Table 2). Three patients had one or more vertebral fractures in the year following the rheumatologic consultation despite the introduction of zoledronic acid or denosumab for two of them. Of the three patients who developed an operative site infection, two had osteopenia.

\section{Discussion}

Prior to corrective surgery of the lumbar spine, osteoporosis and osteopenia were detected in $14.3 \%$ and $42.9 \%$ of cases, respectively, in a single-center population of 28 patients over the age of 50 years for whom a status assessment was requested. After assessment, vitamin D/ calcium supplementation and osteoprotective treatment were prescribed in $71.4 \%$ and $17.8 \%$ of cases, respectively. After surgery, three patients had vertebral osteoporotic fractures, while four other patients had loosening of their implanted device.

Osteoporosis is observed in $21.2 \%$ of women in the 50 - to 84-year age group, ${ }^{6}$ representing a higher prevalence than that of the patients in our population with a significant risk of falling. According to James et al, osteoporosis is present in $8.4 \%$ of patients waiting for hip or knee arthroplasty. ${ }^{7}$ To our knowledge, no work has investigated the prevalence of osteoporosis before corrective surgery of the lumbar spine. During follow-up, we observed a prevalence of $25 \%$ of vertebral fractures or loosening of implanted devices. In the absence of assessment of bone status, it is not excluded that the impact would have been greater. None of our patients were treated with teriparatide, and only one patient was treated with denosumab because the use of these treatments was restricted in France (at least two vertebral fractures for the first, 3 months of bisphosphonate use in the previous year for the second). However, administration of teriparatide resulted in a significant increase in bone mineral density in 17 patients who had total knee arthroplasty. ${ }^{8}$ In addition, in another work, denosumab versus placebo reduced the area of peri-prosthetic osteolysis. ${ }^{9}$ In addition, a TBS $<1.2$ reflects a poor structural state of bone microarchitecture. ${ }^{10}$ In our population, $47 \%$ of patients were below this threshold. However, it is not a threshold for therapeutic intervention, even though TBS may predict the occurrence of osteoporotic vertebral fracture regardless of densitometric results in postmenopausal white Europeans without osteoporosis. ${ }^{10}$ In practice, in a population $>50$ years who must benefit from corrective spine surgery, the value of TBS and the search for risk factors for falls should be taken into account more. 


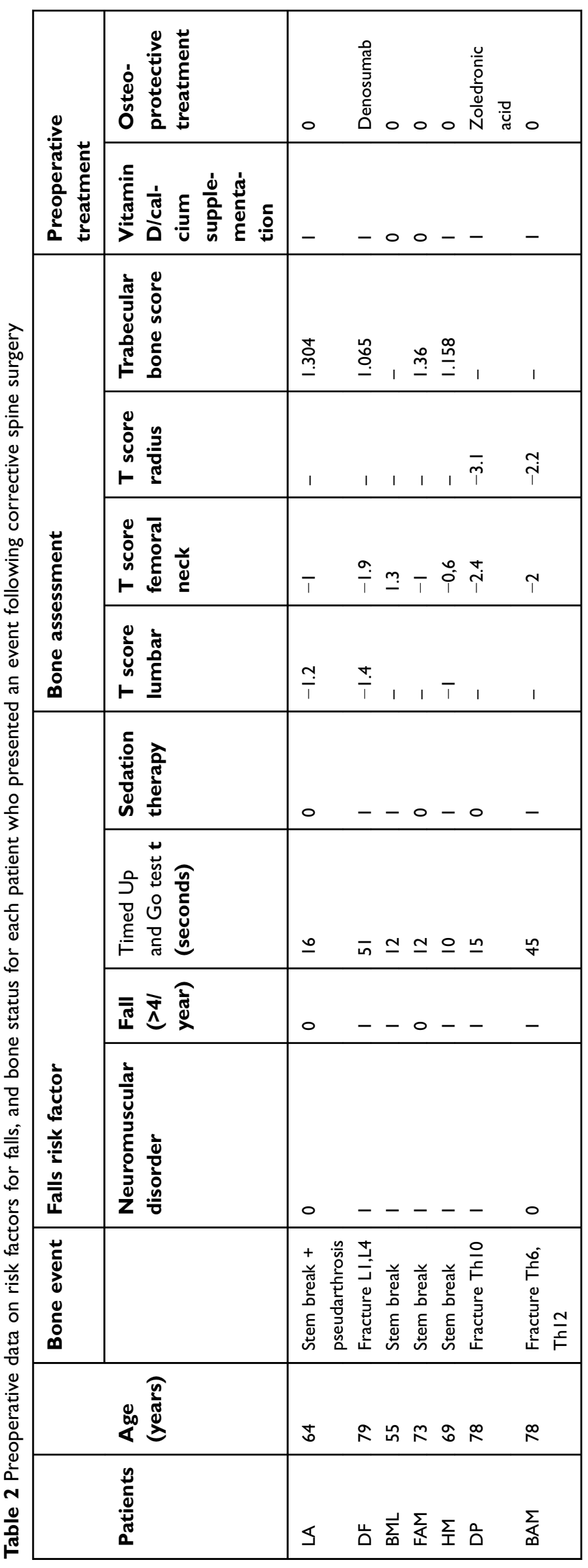

Regarding the occurrence of infection after spine surgery, a Chinese study found osteoporosis as a risk factor for infection of the operative site, with the hypothesis of poor fixation of the implanted device in the vertebra but also a more complex operating procedure (bleeding and longer operative time). ${ }^{11}$

There are two main limits in this study. First, this study was performed in a population of limited size since among the 28 patients included, only 13 of them have benefited from a surgical intervention. Second, patients of this study have been referred to rheumatology consultation for assessment of bone status prior to corrective surgery, which means they had probably more risk factors of osteoporosis and/or of falls.

\section{Conclusion}

Although the number of patients included is low, this is, to the best of our knowledge, the first study to assess both the quantitative and qualitative (bone texture) bone status of an active line of patients over the age of 50 years who had corrective lumbar spine surgery. A quarter of our patients presented a complication related to bone fragility, even though densitometric data were rather reassuring, leading only to low eligibility for osteoprotective treatment. In contrast, our population had risk factors for osteoporotic fractures, including falls and a structural state of altered bone microarchitecture, suggesting the superiority of TBS as an indicator of bone status prior to surgery of the lumbar spine, but a study on a larger population is needed to confirm this hypothesis.

\section{Data availability}

Data are available from the corresponding author for 5 years after publication. Send requests to: totobanse@orange.fr.

\section{Acknowledgments}

The authors are grateful to Nikki Sabourin-Gibbs, Rouen University Hospital, for her help in editing the manuscript.

No financial support or other benefits from commercial sources were received for the work reported in the manuscript.

\section{Disclosure}

Alexis Perez reports a link with MEDICREA, but that does not relate to this work. The authors declare that they have no conflicts of interest in relation to this article.

\section{References}

1. Curran D, Maravic M, Kiefer P, Tochon V, Fardellone P. Epidemiology of osteoporosis-related fractures in France: a literature review. Joint Bone Spine. 2010;77(6):546-551. doi:10.1016/j.jbspin.2010.02.006 
2. Oresanya LB, Lyons WL, Finlayson E. Preoperative assessment of the older patient: a narrative review. JAMA. 2014;311(20):2110-2120. doi:10.1001/jama.2014.4573

3. Bégué T, Adam P, Fessy MH, et al. Periprosthetic fractures around total hip and knee arthroplasty. Introduction and study objectives. Rev Chir Orthop Reparatrice Appar Mot. 2006;92(5):2S34-2S36.

4. Briot K, Cortet B, Thomas T, et al. 2012 update of French guidelines for the pharmacological treatment of postmenopausal osteoporosis. Joint Bone Spine. 2012;79(3):304-313. doi:10.1016/j. jbspin.2012.02.014

5. Kanis JA, Johnell O, Oden A, Johansson H, McCloskey E. FRAX and the assessment of fracture probability in men and women from the UK. Osteoporos Int. 2008;19(4):385-397. doi:10.1007/s00198-007-0543-5

6. Kanis JA, Johnell O, Oden A, Jonsson B, De Laet C, Dawson A. Risk of hip fracture according to the World Health Organization criteria for osteopenia and osteoporosis. Bone. 2000;27(5):585-590.

7. James SJ, Mirza SB, Culliford DJ, Taylor PA, Carr AJ, Arden NK. Baseline bone mineral density and boneturnover in pre-operative hip and knee arthroplasty patients. Bone Joint Res. 2014;3(1):14-19. doi:10.1302/2046-3758.31.2000218
8. Suzuki T, Sukezaki F, Shibuki T, Toyoshima Y, Nagai T, Inagaki K. Teriparatide administration increases periprosthetic bone mineral density after total knee arthroplasty: a prospective study. J Arthroplasty. 2018;33(1):79-85. doi:10.1016/j.arth.2017.07.026

9. Sköldenberg O, Rysinska A, Eisler T, Salemyr M, Bodén H, Muren O. Denosumab for treating periprosthetic osteolysis; study protocol for a randomized, double-blind, placebo-controlled trial. $B M C$ Musculoskelet Disord. 2016;17:174. doi:10.1186/s12891-016-1134-4

10. Boutroy S, Hans D, Sornay-Rendu E, Vilayphiou N, Winzenrieth R, Chapurlat R. Trabecular bone score improves fracture risk prediction in non-osteoporotic women: the OFELY study. Osteoporos Int J. 2013;24:77-85. doi:10.1007/s00198-012-2188-2

11. Lai Q, Song Q, Guo R, et al. Risk factors for acute surgical site infections after lumbar surgery: a retrospective study. J Orthop Surg Res. 2017;12(1):116. doi:10.1186/s13018-017-0612-1.

\section{Publish your work in this journal}

Open Access Rheumatology Research and Reviews is an international, peer-reviewed, open access journal publishing original research, reports, editorials, reviews and commentaries on all aspects of clinical and experimental rheumatology in the clinic and laboratory including the following topics: Pathology, pathophysiology of rheumatological diseases; Investigation, treatment and management of rheumatological diseases; Clinical trials and novel pharmacological approaches for the treatment of rheumatological disorders. The manuscript management system is completely online and includes a very quick and fair peer-review system, which is all easy to use. Visit http://www.dovepress.com/testimonials.php to read real quotes from published authors. 